\title{
In Vitro Cow's Milk Protein-Specific Inflammatory and Regulatory Cytokine Responses in Preterm Infants With Necrotizing Enterocolitis and Sepsis
}

\author{
ADEL E. ABDELHAMID, SHU-LING CHUANG, PETER HAYES, AND JOHN M. E. FELL \\ Divisions of Paediatrics, Obstetrics and Gynaecology [A.E.A., S.-L.C., J.M.E.F.] and Investigative Sciences [P.H.], Imperial College, \\ London SW10 9NH, United Kingdom
}

\begin{abstract}
Enteral feeding with cow's milk formula is associated with neonatal necrotizing enterocolitis (NEC) and sepsis. Dietary antigen sensitization may play a role in promoting and/or sustaining inflammation in both conditions. Aiming at investigating cow's milk protein (CMP)-specific cytokine responses in preterm infants with NEC and sepsis, 14 babies with NEC, 14 matched healthy controls, and 10 septic controls were recruited. Unstimulated and stimulated peripheral blood mononuclear cells (PBMCs) secreting IFN- $\gamma$, IL-4, IL-10, and TGF- $\beta 1$ were counted by the single-cell enzyme-linked immunospot (ELISPOT) assay. During the acute phase of NEC, patients showed a general pattern of a high level of cytokine secretion both when unstimulated and stimulated by mitogen [phytohaemagglutinin (PHA)] and CMPs: beta-lactoglobulin $(\beta-\lg )$ and casein. These responses were more marked to $\beta$-lg for IFN- $\gamma$, IL- 4 , and IL-10 than TGF- $\beta 1$. Cytokine responses in sepsis were lower than in NEC (lowest in healthy controls, with a minimal TGF- $\beta 1$ response). At term, lower frequencies of cytokine-secreting cells were elicited than during the acute phase, except for TGF- $\beta 1$ secreting cells, which increased at term (in response to PHA and CMPs) particularly following not only NEC but also sepsis.

(Pediatr Res 69: 165-169, 2011)
\end{abstract}

$\mathrm{N}$ eonatal necrotizing enterocolitis (NEC) and sepsis are among the more commonly encountered complications in the NICU (1). In both conditions, the disease incidence is inversely related to the birth weight and GA (2). NEC represents a heterogeneous group of intestinal illnesses that encompasses a spectrum of clinical presentations and varying degrees of intestinal inflammation (3). The pathogenesis of NEC is recognized to be multifactorial but remains incompletely understood. Several predisposing factors have been identified including prematurity, formula milk feeding, intestinal hypoxia/ischemia, and bacterial translocation. NEC typically occurs in preterm infants (4) who are also particularly susceptible to sepsis (5).

Most cases of NEC occur in enterally fed infants. The nature of the enteral feed seems to be an important pathogenic factor. Epidemiological studies have suggested an increased risk of NEC in cow's milk formula-fed infants when compared with those receiving breast milk (maternal or donor) (6). To date, the potential link between NEC and formula milk has mostly been explored through identifying the immunoprotec-

Received July 22, 2010; accepted September 9, 2010

Correspondence: Adel E. Abdelhamid, Neonatal Unit, University College of London Hospital, London NW1 2BU, United Kingdom; e-mail: adelabdelhamid@yahoo.co.uk tive elements in breast milk and the determining effect of milk type (formula or breast) on the development of the intestinal microbiota (7). Beta-lactoglobulin $(\beta$-lg), not known to normally exist in the human breast milk, represents $\sim 8.5 \%$ of the protein composition of cow's milk proteins (CMPs), whereas caseins, presenting $<30 \%$ of the human breast milk proteins, constitutes $>80 \%$ of the CMPs (8).

Although the immune system of preterm has been regarded as relatively "immature" compared with that of full term infants, the preterm babies can appropriately respond to antigenic stimuli. However, little is known about the immunological responses of these infants to dietary antigens (9), either in "healthy" state or, perhaps more importantly, in disease states that may involve the mucosal gut barrier such as NEC and sepsis.

We previously demonstrated evidence of sensitization to CMPs in babies with NEC where the in vitro stimulation of peripheral blood mononuclear cells (PBMCs) with casein and $\beta$-lg induced an effector response of both $\mathrm{T}$ helper (Th) cell types: Th1 (IFN- $\gamma$ ) and Th2 (IL-4 and 5) (10).

In this study, by using the sensitive enzyme-linked immunospot (ELISPOT) method that permits the detection of cytokine secretion at a single cell level, we further examined this phenomenon. Infants with NEC, healthy controls, and infants with sepsis were evaluated. In addition to reinvestigating the secretion of IFN- $\gamma$ and IL-4, we also characterized that of the regulatory cytokines IL-10 (secreted by Th regulatory cells type 1 ) and TGF- $\beta 1$ (secreted by Th3) by PBMCs. Furthermore, we repeated all the assessments at term when the infants have recovered from NEC and sepsis compared with healthy controls to measure the changes in cytokines responses over time.

\section{SUBJECTS AND METHODS}

Subjects. Thirty-eight preterm infants admitted to the NICU at Chelsea \& Westminster Hospital between February 2006 and August 2007 were recruited into three study groups: 14 preterm infants with NEC [modified Bell's staging (11) grade II: eight cases and grade III: six cases] who were consec-

Abbreviations: $\boldsymbol{\beta}$-lg, beta-lactoglobulin; CMP, cow's milk protein; ELISPOT, enzyme-linked immunospot; MAb, monoclonal antibody; NEC, necrotizing enterocolitis; PBMC, peripheral blood mononuclear cell; PCA, postconceptional age; PHA, phytohaemagglutinin; PNA, postnatal age; SFC, spot forming cell; $\mathbf{T h}$, T helper cell 
utively identified and recruited within $24 \mathrm{~h}$ of diagnosis; 14 healthy controls with no history of NEC or sepsis who were individually matched by parallel selection for postconceptional age (PCA) and postnatal age (PNA) against the patients with NEC; and 10 infants with late onset ( $>1$ wk of age) blood culture positive sepsis (septic controls), of matched, although not individually, PCA and PNA to the patients with NEC and healthy controls.

Isolation of PBMCs. Approximately $0.5 \mathrm{~mL}$ of heparinized blood was collected from infants in the three groups at diagnosis and then at term. PBMCs were isolated by density gradient centrifugation using standard procedures. Cells were washed in complete medium (R10) containing RPMI 16407 (Sigma Chemical Co.-Aldrich, Gillingham, United Kingdom), 50 $\mathrm{U} / \mathrm{mL}$ penicillin, $50 \mu \mathrm{g} / \mathrm{mL}$ streptomycin, $10 \mu \mathrm{g} / \mathrm{mL}$ gentamicin, $2 \mathrm{mM}$ glutamine, sodium pyruvate, $10 \mathrm{mmol}$ 4-(2-hydroxyethyl)-1-piperazineethanesulfonic acid (HEPES) buffer, and $10 \%$ heat inactivated FCS. The PBMC concentration was determined using a Z1 particle counter (Beckman Coulter, High Wycombe, United Kingdom). The percentage of viable cells was determined by trypan blue exclusion.

Plate coating with capture antibodies. A nitrocellulose-bottomed microtiter plate (MAIPS 4510; Millipore, Watford, United Kingdom) was prewetted with $20 \mu \mathrm{L} /$ well of $70 \%$ ethyl alcohol for $5 \mathrm{~min}$ at room temperature. Alcohol was decanted, and wells were washed three times with $200 \mu \mathrm{L}$ PBS and coated with cytokine-specific monoclonal capture antibody in $100 \mu \mathrm{L}$ of PBS ( $1 \mu \mathrm{g} / \mathrm{well}$ for IFN- $\gamma$, IL-4, and IL-10 and $0.3 \mu \mathrm{g} / \mathrm{well}$ for TGF- $\beta 1)$. The plate was incubated for at least overnight and up to $1 \mathrm{wk}$ at $4^{\circ} \mathrm{C}$ in a sealed bag. Just before use, unadsorbed antibody was decanted, and wells were washed three times with PBS and blocked with $200 \mu \mathrm{L} /$ well of R10 for $2 \mathrm{~h}$ at $37^{\circ} \mathrm{C}$ in $5 \% \mathrm{CO}_{2}$-humidified atmosphere.

Incubation of PBMCs. Blocking medium was decanted and PBMCs added at $0.5 \times 10^{5}$ cells per well in triplicate for each stimulus. Cells were incubated for $20-24 \mathrm{~h}$, in $5 \% \mathrm{CO}_{2}$-humidified atmosphere, at $37^{\circ} \mathrm{C}$ in the absence or presence of respective antigens or mitogens: Keyhole limpet hemocyanin (KLH; $500 \mu \mathrm{g} / \mathrm{mL}), \beta-\lg (500 \mu \mathrm{g} / \mathrm{mL})$, casein $(500 \mu \mathrm{g} / \mathrm{mL})$, and phytohaemagglutinin (PHA; $10 \mu \mathrm{g} / \mathrm{mL}$; Sigma Chemical Co.-Aldrich).

Detection of cytokine-secreting cells. Wells were washed six times with PBS containing 0.05\% Tween 20 (200 $\mu \mathrm{L} /$ well; Sigma Chemical Co.). A $0.4-\mu \mathrm{m}$ filtered biotinylated cytokine-specific monoclonal antibody (MAb) was added in $100-\mu \mathrm{L}$ volumes/well $(0.1 \mu \mathrm{g} / \mathrm{well}$, diluted in PBS/0.5\% BSA) and incubated for $4 \mathrm{~h}$ at room temperature.

Capture and detection MAbs to IFN- $\gamma$ and IL-4 were obtained from MAbtech AB (Nacka Strand, Sweden), whereas those to IL-10 from BD Biosciences (San Diego, CA) and those to TGF- $\beta 1$ (capture Ab: recombinant Human TGF- $\beta$ sRII/Fc Chimera and biotinylated anti-TGF- $\beta 1$ antibody) were obtained from R\&D systems (United Kingdom). At the end of the incubation period, the wells were washed six times with PBS/0.05\% Tween 20 and $100 \mu \mathrm{L}$ avidin-biotin-peroxidase-complex (ABC; Vector Laboratories, Burlingame, CA), prepared per manufacturer's instructions, was added for $1-2 \mathrm{~h}$ at room temperature. The wells were washed three times with $200 \mu \mathrm{L}$ PBS/Tween 20, followed by three times with $200 \mu \mathrm{L}$ PBS and finally, $100 \mu \mathrm{L}$ of amino-ethyl-carbazole (AEC) substrate (Sigma Chemical Co.-Aldrich) were added per well and spot developed at room temperature for 4 min before washing with tap water and drying overnight. Spots were enumerated with an ELISPOT plate reader (Carl Zeiss, Welwyn Garden City, United Kingdom). The final results were expressed as spot forming cells (SFCs) per $10^{5}$ PBMC.

Statistics. The data in each of the three groups were tested for normality of distribution and found to be nonparametric. Different sets of analyses examined the four cytokine-response differences in each group both at the acute stage and at term. Results are expressed as the median and interquartile range (IQR) for each group or as a number and percentage. Comparisons between infants with NEC and healthy controls were analyzed as paired data (Wilcoxon matched-pairs test) as for comparisons between term and acute samples. Comparisons with septic infants were not paired (Mann-Whitney test). The level of significance was set to $1 \%$; only $p \leq 0.01$ were considered to be statistically significant.

Ethical approval. The approval for the study was obtained from the Riverside Research Ethics Committee. Written informed consent was obtained from the parents in each case.

\section{RESULTS}

Demographics. According to the study design, the infants with NEC and healthy control infants were individually matched for PCA and PNA (median PCA, 34.5 wk; median PNA, 32 d). There was no significant difference in age among the three groups although the "septic controls" tended to be younger (median PCA, 28.5 wk; median, PNA 14 d). However, there was a significant difference in birth weight, with the healthy controls significantly heavier than the babies in the other two groups (Table 1).

All the "septic controls" had positive blood cultures (coagulase negative Staphylococci in 8/10). Positive blood cultures were obtained from four infants with NEC (Table 1). C-reactive protein (CRP) was increased in all NEC and septic cases but more for NEC (median CRP, $182 \mathrm{mg} / \mathrm{L}$ versus $104 \mathrm{mg} / \mathrm{L}$; $p=0.02)$.

All infants with NEC and 9 of 10 infants with sepsis had been exposed to cow's milk formula before diagnosis. Six of 14 healthy controls had been exposed to cow's milk formula (Table 1).

Clinical progress until term. After recruitment, babies were followed up until term. Of the patients with NEC, nine underwent laparotomy, with stoma formation in six, and, at term, four were receiving special formula (hydrolyzed or amino acid-based); and two were exclusively receiving breast milk. Among the septic group, two were exclusively receiving breast milk, and eight were receiving formula milk at term.

Two infants with NEC died because of multiorgan failure before term, and hence, in parallel, two healthy controls were dropped from the study at term. Of 12 healthy controls studied at term, five were exclusively fed with breast milk (Table 1).

ELISPOTs were performed for four cytokines (IFN- $\gamma$, IL-4, IL-10, and TGF $\beta 1$ ) on PBMCs from the three groups (NEC, septic, and healthy controls) at presentation and also at term. The results are expressed as number of SFCs per $10^{5}$ mononuclear cells (MNCs). When in vitro stimulation was performed, the response to stimulation is presented as $\Delta$ ELISPOTs. This represents the number of SFCs postantigen/mitogen stimulation minus the number of spontaneously (unstimulated) SFCs.

Spontaneous PBMC ELISPOTs. In healthy preterm infants, there were low numbers of cells spontaneously secreting IFN- $\gamma$, IL- 4 , IL-10, and TGF- $\beta 1$, both in the acute-stage sample and at term. At presentation, significantly higher level of IFN- $\gamma$, IL-4, and IL-10 secreting cells were detected in patients with NEC and sepsis (NEC $>$ sepsis; $p<0.002$ ). This effect was less marked for TGF- $\beta 1$ (NEC $>$ sepsis; $p<$ 0.003). However, at term, the number of IFN- $\gamma$, IL-4, and IL-10 secreting cells had decreased significantly for NEC and sepsis $(p<0.006$ and 0.005 , respectively), whereas TGF- $\beta 1$ secreting cell numbers had increased $(\approx 5$ fold; $p<0.002$ for NEC and 0.005 for sepsis; Table 3).

Response to mitogen stimulation. In vitro stimulation of PBMCs with PHA produced a significant increase in IFN- $\gamma$, IL-4, IL-10, and TGF- $\beta 1$ secreting cells in healthy controls from the acute-stage sample and at term. At presentation, the number of cytokine-secreting cells detected in the NEC group exceeded that of controls (3-9 fold; $p<0.002$ ) for the four cytokines, whereas, in the patients with sepsis, the augmentation of cytokine-secreting cell numbers was less marked $(\approx 2$ fold). At term, the cytokine responses in NEC and septic cases to PHA stimulation were less marked for IFN- $\gamma$, IL-4, and IL-10, although high levels persisted for TGF- $\beta 1$ (Table 2).

Antigen-specific response to $\beta$-lg. $\beta$-lg stimulation elicited small responses in healthy controls both at the initial sample 
Table 1. Demographic feeding and clinical characteristics of babies in the three groups: patients with NEC, septic controls, and healthy controls, at birth, at disease presentation (healthy controls age matched for NEC cases), and at term

\begin{tabular}{|c|c|c|c|}
\hline & Patients with NEC & Septic controls & Healthy controls \\
\hline \multicolumn{4}{|l|}{ At birth } \\
\hline Number & 14 & 10 & 14 \\
\hline Males: females & $10: 4$ & $7: 3$ & $8: 6$ \\
\hline PCA (wk) [Median (range)] & $27(24-35)$ & $26.5(24-33)$ & $27(24-35)$ \\
\hline Weight (g) [Median (range)] & $845(560-2400)$ & $833(544-1800)$ & $1042(715-3086)$ \\
\hline \multicolumn{4}{|c|}{$\begin{array}{l}\text { At presentation (acute stage or acute-stagematched) } \\
\text { sampling point }\end{array}$} \\
\hline Number & 14 & 10 & 14 \\
\hline PCA (wk) [Median (range)] & $32.5(29-36)$ & $28.5(28-35)$ & $32.5(29-36)$ \\
\hline PNA (d) [Median (range)] & $32(3-61)$ & $15(10-46)$ & $32(3-61)$ \\
\hline Weight (g) [Median (range)] & $1305(840-2870)$ & $884(640-1860)$ & $1523(740-3000)$ \\
\hline \multicolumn{4}{|l|}{ Feeding } \\
\hline Exclusively breast milk & 0 & 1 & 8 \\
\hline Formula or mixed [n $(\%)]$ & $14(100)$ & $9(90)$ & $6(43)$ \\
\hline \multirow[t]{6}{*}{ Blood culture $[\mathrm{n}(\%)]$} & Positive: 4 (28) & Positive: 10 (100) & - \\
\hline & E. coli: $1(7)$ & Staphylococcus aureus: 1 (10) & \\
\hline & Klebsiella: 1 (7) & Bacillus cereus: 1 (10) & \\
\hline & CONS 1 (7) & CONS: $8(80)$ & \\
\hline & Multiresistant & & \\
\hline & Staphylococcus aureus: 1 (7) & & \\
\hline \multicolumn{4}{|l|}{ At term } \\
\hline Number & 12 & 10 & 12 \\
\hline Males: females & $8: 4$ & $7: 3$ & $6: 6$ \\
\hline \multicolumn{4}{|c|}{ Median (range) [Median (range)] } \\
\hline PCA (wk) [Median (range)] & $37(37-39)$ & $37(37-37)$ & $37(37-37)$ \\
\hline PNA (d) [Median (range)] & $71(31-93)$ & $77(28-91)$ & $60(16-77)$ \\
\hline Weight (g) [Median (range)] & $1950(1250-2690)$ & $2255(1988-2900)$ & $2500(1800-3200)$ \\
\hline \multicolumn{4}{|l|}{ Feeding } \\
\hline Exclusively breast milk & 2 & 2 & 5 \\
\hline Standard formula & 6 & 8 & 7 \\
\hline Special formula & 4 & 0 & 0 \\
\hline
\end{tabular}

Table 2. Frequencies of PBMCs spontaneously secreting cytokines in patients with NEC, septic controls, and healthy controls (ELISPOT)

\begin{tabular}{|c|c|c|c|c|c|}
\hline \multirow[b]{2}{*}{ Group } & \multirow[b]{2}{*}{ Sample point } & \multicolumn{4}{|c|}{ Cytokine ELISPOTs (SFC/10 5 PBMCs) } \\
\hline & & IFN- $\gamma$ & IL-4 & IL-10 & TGF- $\beta 1$ \\
\hline \multirow[t]{3}{*}{ Patients with NEC } & Acute & $41(30,52)$ & $28(14,41)$ & $21(15,31)$ & $7.5(6,10)$ \\
\hline & Term & $11(7,18)$ & $8(5,14)$ & $10(8,17)$ & $43(34,56)$ \\
\hline & & $p=0.006$ & $p=0.01$ & $p=0.09$ & $p=0.002$ \\
\hline \multirow[t]{3}{*}{ Septic controls } & Acute & $21(10,28)$ & $13(9,20)$ & $10.5(9,16)$ & $5(5,7)$ \\
\hline & Term & $6.5(4,9)$ & $5.5(3,9)$ & $5.5(4.5,7)$ & $32(21,42)$ \\
\hline & & $p=0.005$ & $p=0.005$ & $p=0.005$ & $p=0.005$ \\
\hline \multirow[t]{3}{*}{ Healthy controls } & Acute & $4(2,15)$ & $7.5(4,10)$ & $6(4,10)$ & $3.5(2,8)$ \\
\hline & Term & $2.5(1,8)$ & $4.5(2,6)$ & $2.5(1,5)$ & $5.5(3,10.5)$ \\
\hline & & $p=0.01$ & $p=0.002$ & $p=0.002$ & $p=0.15$ \\
\hline
\end{tabular}

Values represent median and IQRs of SFCs frequencies. The $p$ value refers to the level of statistical significance of the difference between the acute stage (at presentation) and the term results for each group (Wilcoxon matched-pairs test).

Table 3. Increased frequencies of PBMCs secreting cytokines in patients with NEC, septic controls, and healthy controls ( $\triangle E L I S P O T)$ after stimulation with $P H A$

\begin{tabular}{|c|c|c|c|c|c|}
\hline \multirow[b]{2}{*}{ Group } & \multirow[b]{2}{*}{ Sampling point } & \multicolumn{4}{|c|}{ Cytokine $\Delta$ ELISPOTs (SFC/10 5 PBMCs) } \\
\hline & & IFN- $\gamma$ & IL-4 & IL-10 & TGF- $\beta 1$ \\
\hline \multirow[t]{3}{*}{ Patients with NEC } & Acute & $250(170,631)$ & $729(618,783)$ & $679(625,767)$ & $112(85,138)$ \\
\hline & Term & $142(97,250)$ & $160(100,427)$ & $183(126,435)$ & $153(104,164)$ \\
\hline & & $p=0.002$ & $p=0.002$ & $p=0.002$ & $p=0.01$ \\
\hline \multirow[t]{3}{*}{ Septic controls } & Acute & $116(102,155)$ & $145(81,187)$ & $143(79,176)$ & $75(47,93)$ \\
\hline & Term & $64(40,75)$ & $77(53,96)$ & $66(42,91)$ & $44(18,86)$ \\
\hline & & $p=0.005$ & $p=0.005$ & $p=0.005$ & $p=0.005$ \\
\hline \multirow{3}{*}{ Healthy controls } & Acute & $87(48,129)$ & $82(40,109)$ & $75(36,84)$ & $36(20,64)$ \\
\hline & Term & $36(21,50)$ & $30(22,72)$ & $30(15,54)$ & $38(26,67)$ \\
\hline & & $p=0.002$ & $p=0.002$ & $p=0.002$ & $p=0.22$ \\
\hline
\end{tabular}

Values represent the increased median and IQRs of increase in SFC frequencies ( $\triangle \mathrm{SFCs}$ ). The $p$ value refers to the level of statistical significance of the difference between the acute stage (at presentation) and the term results for each group (Wilcoxon matched-pairs test). 

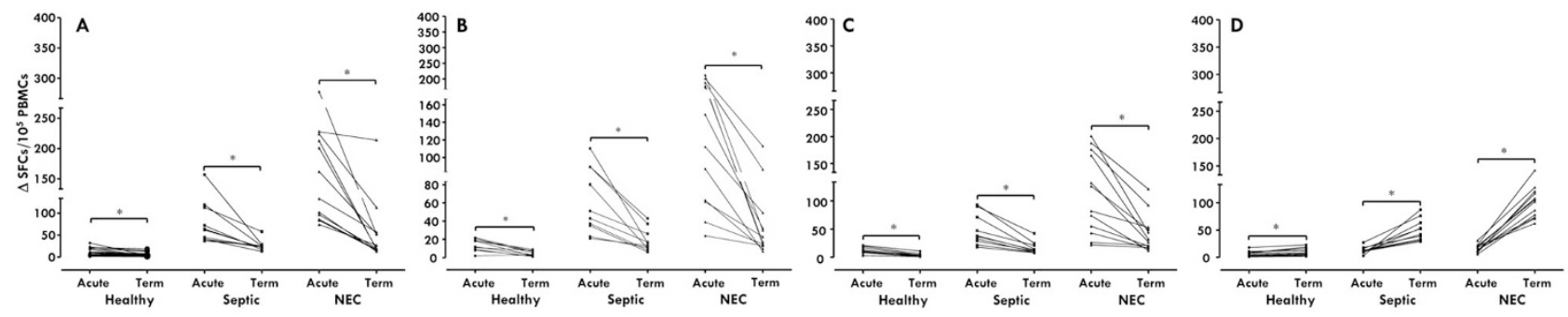

Figure 1. The increased frequencies of PBMCs-secreting cytokines in patients with NEC, septic controls, and healthy controls ( $\triangle$ ELISPOT) after stimulation with $\beta$-lg at presentation (acute) and at term (term). The frequencies of IFN- $\gamma(A)$, IL-4 (B), IL-10 $(C)$, and TGF- $\beta 1(D)$ secreting cells are expressed as $\Delta$ SFCs per $10^{5}$ MNCs ( $\triangle$ SFC per $\left.10^{5} \mathrm{PBMCs}\right)$, which represents the difference between average numbers of spots in the antigen-treated wells and those in the unstimulated wells at each stage of sampling. Each line represents a single subject. $* p<0.01$ that refers to a level of statistical significance of the different frequencies between the two time points for each group (Wilcoxon matched-pairs test).
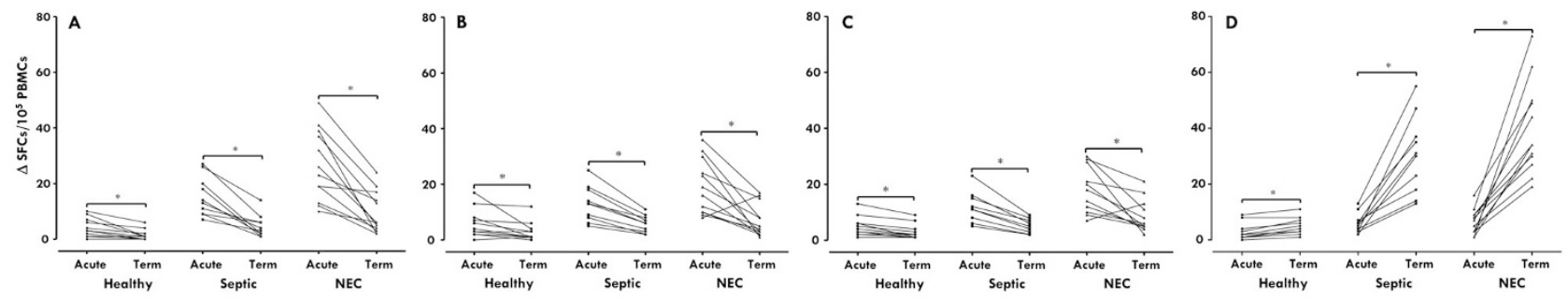

Figure 2. The increased frequencies of PBMCs-secreting cytokines in patients with NEC, septic controls, and healthy controls ( $\triangle$ ELISPOT) after stimulation with casein, at presentation (acute) and at term (term). The frequencies of IFN- $\gamma(A)$, IL-4 (B), IL-10 (C), and TGF- $\beta 1(D)$ secreting cells are expressed as $\Delta$ SFCs per $10^{5}$ MNCs ( $\triangle$ SFC per $10^{5}$ PBMCs), which represents the difference between average numbers of spots in the antigen-treated wells and those in the unstimulated wells at each stage of sampling. Each line represents a single subject. ${ }^{*} p<0.01$ that refers to a level of statistical significance of the different frequencies between the two time points for each group (Wilcoxon matched-pairs test).

and at term for all the four cytokines. These responses were significantly enhanced in NEC and sepsis (NEC > sepsis; $4-10$ fold; $p<0.001)$ for IFN- $\gamma$, IL-4, and IL-10 at presentation. This enhancement was significantly less marked at term (Fig. 1). However, TGF- $\beta 1$ secretion was only slightly enhanced at presentation (NEC and sepsis, $\approx 2$-fold enhancement; $p=0.007$ ); however, at term, $\beta$-lg-induced enhancement was significantly greater (NEC and sepsis, $>5$-fold enhancement; Fig. 1).

Antigen-specific response to casein. Casein stimulation of PBMCs from patients with NEC and from septic and healthy controls produced a similar pattern of response to $\beta$-lg, both at presentation and at term. Minimal responses were elicited in healthy controls (both initial and term samples) for all the four cytokines. These responses were enhanced in NEC and sepsis (NEC $>$ sepsis, $3-5$ fold; $p<0.002$ ) for IFN- $\gamma$, IL-4, and IL-10 at presentation. This enhancement was significantly less marked at term (Fig. 2). However, TGF- $\beta 1$ secretion was only marginally enhanced in the acute stage for NEC and sepsis; however, at term, casein-induced enhancement was significantly greater (NEC and sepsis, $>5$-fold enhancement; Fig. 2). Stimulation with KLH induced negligible responses in all samples (NEC, sepsis, and healthy controls at presentation and at term for the four cytokines assessed).

\section{DISCUSSION}

In this study, we have used the sensitive ELISPOT assay to measure in vitro the frequency of PBMCs secreting four cytokines (IFN- $\gamma$, IL-4, IL-10, and TGF- $\beta 1$ ) in preterm infants with neonatal NEC and sepsis during the acute stage of the disease and at term compared with "healthy" controls of matched gestation and PNA. We present further evidence of CMP ( $\beta-\lg$ and casein) sensitization in preterm infants with NEC, and a similar, yet less pronounced, response in septic preterm babies, during the acute stage of the disease. Furthermore, we also characterized the natural history of the observed responses in the three groups at term.

In line with our previous observations (10), we found, during the acute stage of NEC, an effector response to milk proteins of both Th1 (IFN- $\gamma$ ) and Th2 (IL-4) cytokine profiles. We also showed a concomitant secretion of regulatory cytokines mainly of IL-10 and, to a much lesser extent, of TGF$\beta 1$. Similar, yet less marked, effects were also observed in the preterm infants with sepsis.

Reviewing the feed histories of the studied infants, nearly all the infants with NEC and sepsis (23/24) had been exposed to cow's milk formula, and only one was exclusively breast fed. In the healthy controls, many infants had also been exposed to cow's milk formula (6/14). Thus, it is unlikely that the in vitro sensitization observed in this study is because of the differences in previous dietary antigen exposure as a result of the type of milk feeds received. Changes in mucosal permeability to dietary antigens (CMPs in this case) would represent a plausible mechanism for sensitization in infants with NEC, because mucosal damage is a characteristic of the condition. Such a mechanism is also plausible in infants with sepsis because a number of the systemic inflammatory mediators found in sepsis can also increase mucosal permeability (12). 
At term, after recovery from NEC and/or sepsis, the sensitization profile changes significantly with a decline in IFN- $\gamma$, IL-4, and also IL-10 responses. The decline in these milk protein specific responses with clinical improvement mirrors the decrease in background cell reactivity at rest and following stimulation with PHA. After NEC, the infants were initially refed, in accordance with our unit practice, using either breast milk or a hypoallergenic formula (hydrolyzed CMP or amino acid formula), if breast milk is not available. At term, $\sim 62 \%$ of the infants in this study were once again receiving standard cow's milk formula. A fall in inflammatory cytokine secretion to $\beta$-lg was observed at term in all the patients with NEC and also in the infants with sepsis. Eighty percent of the infants with sepsis had remained on standard cow's milk formula. The decline in milk protein sensitization at term could thus not be accounted for by the type of milk feeds the infants were receiving at that time. During the acute stage of the disease, the regulatory cytokine (IL-10 and TGF- $\beta 1$ ) responses to provocation by CMPs were less marked and minimal in the case of TGF- $\beta 1$.

Both, IL-10 and TGF- $\beta 1$, play a role in the resolution of inflammation. It is possible that they contribute to the "fine tuning" rather than to the abrogation of inflammatory responses (13). The IL-10 up-regulation observed in all subjects during the acute-stage disease is in contrast to the pattern of significantly lower frequencies of TGF- $\beta 1$-secreting cells, both spontaneously and in response to stimulation by CMPs and PHA.

At term, when the infants had recovered from NEC and sepsis, the TGF- $\beta 1$ responses to milk proteins became more prominent. The time course of this increase in TGF- $\beta 1$ secretion, indicating a change from a proinflammatory (IFN- $\gamma$ and IL-4) toward a counter-inflammatory/regulatory (IL-10 and TGF- $\beta 1$ ) profile, coincides with the reduction in the mucosal inflammation during recovery. Over time, while the effect of sensitization to CMPs abates, "tolerance" is reestablished in these subjects.

TGF- $\beta 1$ has previously been investigated in several intestinal inflammatory conditions $(14,15)$ and is known for its potent effects in mitigating mucosal inflammation, although less is known about its expression during the resolution of sepsis. Animal studies have shown that septic mice with TGF- $\beta 1$ gene deletions develop spontaneous organ failure associated with infiltration of MNCs, which indicates that this gene may play a role in controlling inflammatory responses (16). In addition to its immunologic effects, TGF- $\beta 1$ is also known for its enhancing effect on fibrous tissue formation (17). The relation of this compensatory increased TGF- $\beta 1$ expression to such long-term complications as stricture formation in NEC is worthy of further evaluation.

The similarities in this study between the observations made on the infants with NEC and those with sepsis are striking. It is likely that NEC and sepsis represent a spectrum of disease with overlapping causes and clinical manifestations.
The features described in early NEC (Bell's staging 1) are commonly found in and indistinguishable from infants with septic ileus. The gastrointestinal tract is recognized as a major factor in the development of neonatal sepsis (18). The development of food antigen sensitization in sepsis in preterm infants, which we have observed, further points to a major role of the mucosa and mucosal permeability as a cause or a consequence of systemic sepsis (19).

In conclusion, our data furthers our understanding of cytokine regulation in the process of disease evolution. The observations provide further evidence of CMP sensitization in preterm infants in different disease settings. The clinical implications of the observed in vitro CMP sensitization on the disease process or in the longer term are unclear and will require further epidemiological and immunological studies. However, in clinical practice, many neonatal units have already altered their feed strategies in infants recovering from NEC away from whole protein cow's milk feeds to breast milk, hydrolyzed protein, or amino acid formulations. The potential of modulation of immune responses through dietary antigen manipulation after $\mathrm{NEC}$ also requires further investigation.

\section{REFERENCES}

1. Schnabl KL, Van Aerde JE, Thomson AB, Clandinin MT 2008 Necrotizing enterocolitis: a multifactorial disease with no cure. World J Gastroenterol 14:2142-2161

2. Holman RC, Stoll BJ, Curns AT, Yorita KL, Steiner CA, Schonberger LB 2006 Necrotizing enterocolitis hospitalisations among neonates in the United States. Paediatr Perinat Epidemiol 20:498-506

3. Lin PW, Nasr TR, Stoll BJ 2008 Necrotizing enterocolitis: recent scientific advances in pathophysiology and prevention. Semin Perinatol 32:70-82

4. van Elburg RM, Fetter WP, Bunkers CM, Heymans HS 2003 Intestinal permeability in relation to birth weight and gestational and postnatal age. Arch Dis Child Fetal Neonatal Ed 88:F52-F55

5. Lin PW, Stoll BJ 2006 Necrotizing Enterocolitis. Lancet 368:1271-1283

6. Rees CM, Eaton S, Pierro A 2010 National prospective surveillance study of necrotizing enterocolitis in neonatal intensive care units. J Pediatr Surg 45:13911397

7. Walker A 2010 Breast milk as the gold standard for protective nutrients. J Pediatr 156:S3-S7

8. Crittenden RG, Bennett LE 2005 Cow's milk allergy: a complex disorder. J Am Coll Nutr 24:582S-591S

9. Liem JJ, Kozyrskyj AL, Huq SI, Becker AB 2007 The risk of developing food allergy in premature or low-birth weight children. J Allergy Clin Immunol 119:1203-1209

10. Chuang SL, Hayes PJ, Ogundipe E, Haddad M, MacDonald TT, Fell JM 2009 Cow's milk protein-specific T-helper type I/II cytokine responses in infants with necrotizing enterocolitis. Pediatr Allergy Immunol 20:45-52

11. Walsh MC, Kliegman RM 1986 Necrotizing enterocolitis: treatment based on staging criteria. Pediatr Clin North Am 33:179-201

12. Neu J 2007 Gastrointestinal development and meeting the nutritional needs of premature infants. Am J Clin Nutr 85:629S-634S

13. Thompson C, Powrie F 2004 Regulatory T cells. Curr Opin Pharmacol 4:408-414

14. Ashwood P, Harvey R, Verjee T, Wolstencroft R, Thompson RP, Powell JJ 2004 Functional interactions between mucosal IL-1, IL-1ra and TGF- $\beta 1$ in ulcerative colitis. Inflamm Res 53:53-59

15. Monteleone G, Pallone F, MacDonald TT 2004 Smad7 in TGF- $\beta$-mediated negative regulation of gut inflammation. Trends Immunol 25:513-517

16. Ayala A, Chung CH, Grutkoski PS, Song GY 2003 Mechanisms of immune resolution. Crit Care Med 31:S558-S571

17. Yuen DE, Stratford AF 2004 Vitamin A activation of transforming growth factor- $\beta 1$ enhances porcine ileum wound healing in vitro. Pediatr Res 55:935-939

18. Sharma R, Tepas JJ, Hudak ML, Mollitt DL, Wludyka PS, Teng R-J, Premachandra BR 2007 Neonatal gut barrier and multiple organ failure: role of endotoxin and proinflammatory cytokines in sepsis and necrotizing enterocolitis. J Pediatr Surg 42:454-461

19. Cantani A, Micera M 2005 Neonatal cow's milk sensitization in 143 case-reports: role of early exposure to cow's milk formula. Eur Rev Med Pharmacol Sci 9:227-230 